\title{
Effect of Grip Strength on Controlled Force Exertion in Different Strength Exertion Phases in Young Men
}

\author{
Yoshinori Nagasawa ${ }^{1, *}$, Shinichi Demura ${ }^{2}$ \\ ${ }^{1}$ Department of Health and Sports Sciences, Kyoto Pharmaceutical University, Kyoto, Japan \\ ${ }^{2}$ Graduate School of Natural Science \& Technology, Kanazawa University, Kanazawa, Japan \\ *Corresponding author: ynaga@mb.kyoto-phu.ac.jp
}

Received April 08, 2019; Revised May 17, 2019; Accepted June 13, 2019

\begin{abstract}
It is important to develop a method for the accurate measurement of controlled force exertion (CFE). The CFE test requires subjects to exert force while coordinating submaximal grip strength; therefore, grip strength may affect the CFE value. This study examined the differences in measured values in low and high phases of demand value in the CFE test and the relationship between the measured values and grip strength using 54 healthy young males aged 19-23 years. On the basis of standard values of grip strength related to age (455.7 $\pm 67.6 \mathrm{~N})$, participants were divided into the following three groups: G1, with low grip strength ( $\mathrm{n}=13$, mean age, 19.9 years, standard deviation $(\mathrm{SD})=0.8$ years); $\mathrm{G} 2$, with medium grip strength $(\mathrm{n}=33$, mean age, 20.6 years, $\mathrm{SD}=1.3$ years); and G3, with high grip strength $(\mathrm{n}=8$, mean age, 21.6 years, $\mathrm{SD}=0.7$ years). The participants adjusted the submaximal grip strength of their dominant hands according to changes in the demand values, which were displayed as a sinusoidal waveform with a frequency of $0.1 \mathrm{~Hz}$ on a computer screen. The test, which lasted for $40 \mathrm{~s}$, was performed three times, with one-minute intervals, after one practice trial. The sum of the differences between the demand value and the measured grip exertion value in the low demand value phase [5\%-15\% maximum voluntary contraction (MVC)] and high demand value phase $[15 \%-25 \% \mathrm{MVC}]$ for $30 \mathrm{~s}$ was used as the evaluation parameter. Significant differences were found in the measured CFE values of the three groups only in the low demand value phase $(\mathrm{F}=3.43, \mathrm{p}<0.05)$, and the values for $\mathrm{G} 3$ were lower than those for $\mathrm{G} 1$ and $\mathrm{G} 2$, but the effect size $\left(\eta^{2}\right)$ was low $\left(\eta^{2}=0.12\right)$. The CFE values showed significant low correlation with grip strength only in the high demand value phase $(r=-0.32, p<0.05)$. We inferred that the difference in maximum grip strength has a negligible effect on the measured value in both low and high demand value phases of the CFE test in young males.
\end{abstract}

Keywords: force output, grip strength, psychomotor performance, tracking paradigm, visuomotor processing

Cite This Article: Yoshinori Nagasawa, and Shinichi Demura, "Effect of Grip Strength on Controlled Force Exertion in Different Strength Exertion Phases in Young Men." American Journal of Sports Science and Medicine, vol. 7, no. 2 (2019): 40-44. doi: 10.12691/ajssm-7-2-3.

\section{Introduction}

Precise controlled exertion of force (CFE) is essential to perform daily living activities smoothly. Accurate and efficient movements depend on the precise control of the small muscle groups of the hand and fingers, and both the magnitude and the style of force output are largely affected by neuromuscular function [1]. The dynamic properties of force control are influenced by a wide range of factors, including physical maturation [1,2], aging of neuromuscular pathways [3], and the magnitude of exerted force to demand value [4]. In particular, the voluntary coordinated force exertion ability, i.e., the CFE, plays a key role in achieving skillful and efficient movements requiring feedback information, manual dexterity, and hand-eye coordination [5].

The CFE test evaluates motor control function that involves exertion of muscular force while coordinating a changing demand value [6]. Motor control function is considered to be excellent when muscle contraction and relaxation are both smooth and accurate in accordance with the demand value, i.e., movement with small errors between the demand value and the exerted value [7]. The CFE test can rationally and objectively evaluate grading, spacing (space perception), and timing, which are important elements of CFE [6].

The test requires submaximal grip control, and hand-eye coordination is assessed in test performance; therefore, it is also useful in evaluating neuromuscular function in elderly persons with impaired physical functions [8]. The CFE test typically uses visual tracking paradigms that display varying demand and exerted values as a sinusoidal waveform or as a bar chart $[3,6,9]$.

Kubota and Demura [10] confirmed that the degree of measured value (error between demand and exerted values) differs in the ascending and the decreasing phases in the CFE test. As the degree of exerted grip strength and exerted style (contraction and relaxation of muscle) differ [11], the effect on both phases differs; hence, the CFE values in both phases are also considered to differ. 
Every individual's maximum voluntary contraction (MVC) differs; therefore, the relative value to MVC (5\%-25\% of MVC) [6] is used in the CFE test. For instance, when the subject's MVC is $784 \mathrm{~N}(80 \mathrm{~kg})$, he exerts a grip strength of $39.2 \mathrm{~N}-196 \mathrm{~N}$ (4 kg--8 kg), whereas when his MVC is $392 \mathrm{~N}(40 \mathrm{~kg})$, he exerts a grip strength of $19.6 \mathrm{~N}-98 \mathrm{~N} \mathrm{(2}$ $\mathrm{kg}-10 \mathrm{~kg}$ ). Therefore, the difference of MVC may affect the measured CFE value. However, little attention has been paid to this problem until now.

As stated, because the CFE test uses a submaximal exertion of grip strength, it is a useful test for people of all ages, including children and elderly, and can be used in the rehabilitation of the elderly. However, MVC differs substantially with respect to age [12] and sex [13]. The CFE test uses a sum of errors between the exerted value and demand value as a measured value in repeated ascending and decreasing phases; hence, an individual's measured value is not affected significantly if an error in both phases differs. However, if the MVC affects a measured value, even when the relative value is used, it will affect an individual's CFE evaluation.

Thus, it is necessary to clarify the effect of MVC on the measured value. It is hypothesized that, if MVC affects a measured value, as the degree of demand value in the low demand value (5\%-15\% of MVC) and high demand value (15\%-25\% of MVC) phases differs, both its effect and the relationship of MVC and the measured value differ in both phases.

Using young males with different MVCs, this study examined the difference of measured values in the low demand value and high demand value phases in the CFE test and the relationship between the measured value and the grip strength in both phases.

\section{Methods}

\subsection{Subjects}

Subjects were 54 young male university students (age, $19-23$ years; mean age, 20.5 years; $\mathrm{SD}=1.2$ years; mean height, $173.4 \mathrm{~cm}$; $\mathrm{SD}=5.2 \mathrm{~cm}$; mean weight, $67.1 \mathrm{~kg}$; SD $=7.2 \mathrm{~kg}$ ). All the subjects, except two, were judged to be right-handed based on the inventory by Demura et al. [14]. The mean values of height and body mass were similar to the Japanese normative values at the same age level [15]. No subject reported either previous wrist injuries or upper limb nerve damage, and all were in good health. Before measurements were performed, both the purpose and procedure of the study were explained in detail, and signed informed consent was obtained from all subjects. The Ethics Committee on Human Experimentation of Kyoto
Pharmaceutical University approved this experimental protocol. No subject had undergone a CFE test previously.

\subsection{Apparatus of CFE Measurement}

The size of the grip of the CFE measurement apparatus was set for effective squeezing. Grip strength and CFE tests were measured using a Smedley's handgrip mechanical dynamometer (GRIP-D5101; Takei, Tokyo, Japan), with an accuracy of $\pm 2 \%$ in the range of $0-979.7 \mathrm{~N}$ (output range of $1--3 \mathrm{~V}$ ). The grip strength value was transmitted to a computer at a sampling rate of $10 \mathrm{~Hz}$ through an RS-232C data output cable (Elecom, Tokyo, Japan) following $\mathrm{A} / \mathrm{D}$ conversion with a quantization bit rate of 12 bits (input range of 1-5V). The CFE apparatus has been described in detail previously by Nagasawa \& Demura [6].

\subsection{Measurement of Maximal Grip Strength}

Grip strength of the dominant hand was measured twice, with a 1 min rest interval, in a standing position. All subjects were instructed to exert their greatest possible grip strength with their dominant arm hanging naturally at their side and without touching the grip dynamometer to their body or shaking it. The subjects were given no verbal encouragement. The greater value obtained in the two trials was used as the maximum grip strength value $[6,8]$.

\subsection{Measurement of CFE}

The CFE test was similar to the commonly used grip strength test described above [16,17], except for the involvement of the prolonged submaximal grip exertion $(5 \%-25 \%)$. The grip size during the CFE test was set for effective squeezing, but most subjects used the same grip size during the maximal grip hold exertion test.

In this study, a sinusoidal waveform method was used to correctly measure the sum of the differences between the demand value and the grip exertion value (measured value) in the low demand value phase (5\%-15\% MVC) and the high demand value phase $(15 \%-25 \% \mathrm{MVC})$. The demand and grip strength exertion values were presented on the display screen simultaneously. Degree of the grip exertion value and demand value were displayed as sinusoidal waveforms. The demand value varied over a period of $40 \mathrm{~s}$, with a frequency of $0.1 \mathrm{~Hz}[18,19]$. Subjects stood $70 \mathrm{~cm}$ from the display and wore glasses when required. They tracked the demand value moving from left to right with the waveform change displayed on the computer. The grip strength exertion value also moved from left to right with the waveform change (see Figure 1).

Table 1. Physical characteristics of participants

\begin{tabular}{|c|c|c|c|c|c|c|c|c|c|}
\hline \multirow[t]{2}{*}{ MVC group } & \multirow[t]{2}{*}{$n$} & \multicolumn{2}{|c|}{ Age (years) } & \multicolumn{2}{|c|}{ Height (cm) } & \multicolumn{2}{|c|}{ Body mass (kg) } & \multicolumn{2}{|c|}{ Grip strength (N) } \\
\hline & & $M$ & $S D$ & $M$ & $S D$ & $M$ & $S D$ & $M$ & $S D$ \\
\hline Low & 13 & 19.9 & 0.76 & 170.4 & 5.25 & 65.0 & 10.69 & 363.9 & 23.56 \\
\hline Midium & 33 & 20.6 & 1.32 & 174.6 & 5.02 & 67.2 & 5.88 & 468.2 & 35.14 \\
\hline High & 8 & 21.6 & 0.68 & 173.4 & 4.01 & 70.3 & 3.89 & 562.7 & 51.59 \\
\hline Total & 54 & 20.5 & 1.24 & 173.4 & 5.16 & 67.1 & 7.17 & 457.1 & 71.49 \\
\hline
\end{tabular}




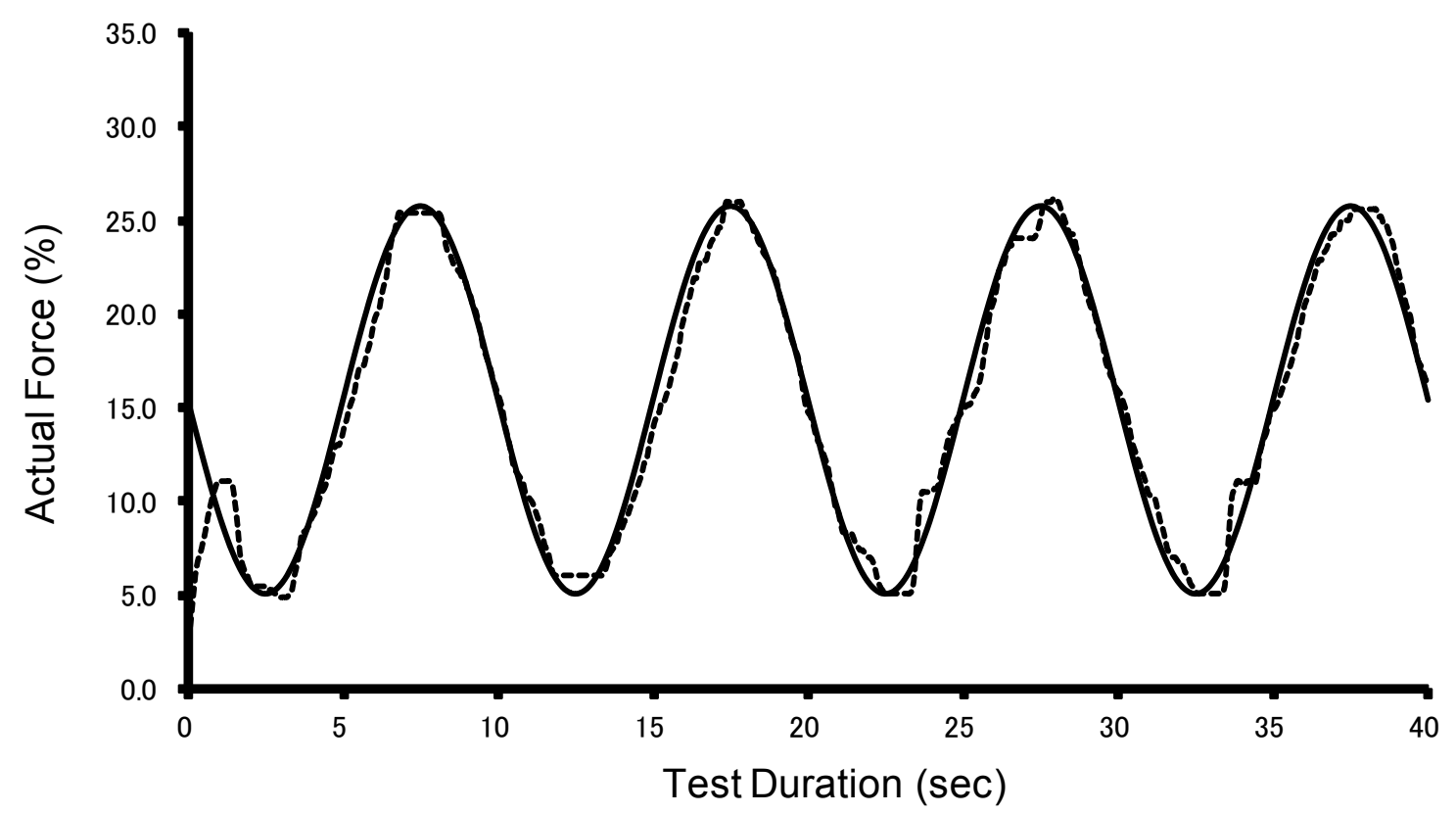

Figure 1. Sinusoidal waveform display $(100 \mathrm{~mm} \times 140 \mathrm{~mm})$ of the demand value. The solid waveform (A) shows the demand value and the broken waveform (B) is the exertion value of grip strength. The test was to fit line B (exertion value of grip strength) to line A (demand value), whice varied in the rang of 5\%--25\% of maximum grip strength. the length of the display is $33 \mathrm{~mm}$ from top to bottom. Frequency of chang in demand value is $0.1 \mathrm{~Hz}$. The test time was $40 \mathrm{~s}$ for each trial. The controlled force exertion was calculated using the data from $30 \mathrm{~s}$ of the trial following the initial $10 \mathrm{~s}$ of the 40 -s period

As the grip strengths of individuals differ, the relative demand value to maximum grip strength was used [18,20]. That is, a software program was designed to display the same demand value, within a constant range, for all subjects on a personal computer. The CFE test was performed using three trials, with one-minute rest intervals, after one practice trial that confirmed whether subjects could perform the CFE test. The duration of each trial was $40 \mathrm{~s}$, and, based on the study of Nagasawa et al. [8] and Nagasawa and Demura [21], the CFE measured values were estimated using the data from the three trials, excluding the first $10 \mathrm{~s}$ of each trial. The sum of the percentages of the differences between the demand values and the grip strength values over 30 -s in both low (5\%-15\% MVC) and high (15\%-25\% MVC) phases was used as an estimate of the CFE measured values [6], with smaller differences indicating better performance. A mean of the results of the second and third trials was used for analysis [20,21].

\subsection{Statistical Analysis}

Data were analyzed using SPSS version 21.0 for Windows software (SPSS Inc., Tokyo, Japan). Using ordinary statistical methods, data were reported as means $(\mathrm{M}) \pm \mathrm{SD}$. Participants were divided into three groups based on grip strength and normative values of the same age (455.7 \pm $67.6 \mathrm{~N}$ ): low (13 participants; mean age, 19.9 years, $\mathrm{SD}=0.8$ years $)$, medium (33 participants; mean age, 20.6 years, $\mathrm{SD}=1.3$ years), and high ( 8 participants; mean age, 21.6 years, $\mathrm{SD}=0.7$ years). One-way analysis of variance was used to examine significant differences in the three groups between CFE mean scores and differing maximal grip strength. When a significant main effect was identified, a multiple comparison test was conducted using Tukey's honestly significant difference method for pairwise comparisons. Correlation analyses were used to identify the relationships between $\mathrm{CFE}$ and maximum grip strength. For all tests, a p-value $<0.05$ was considered to be significant.

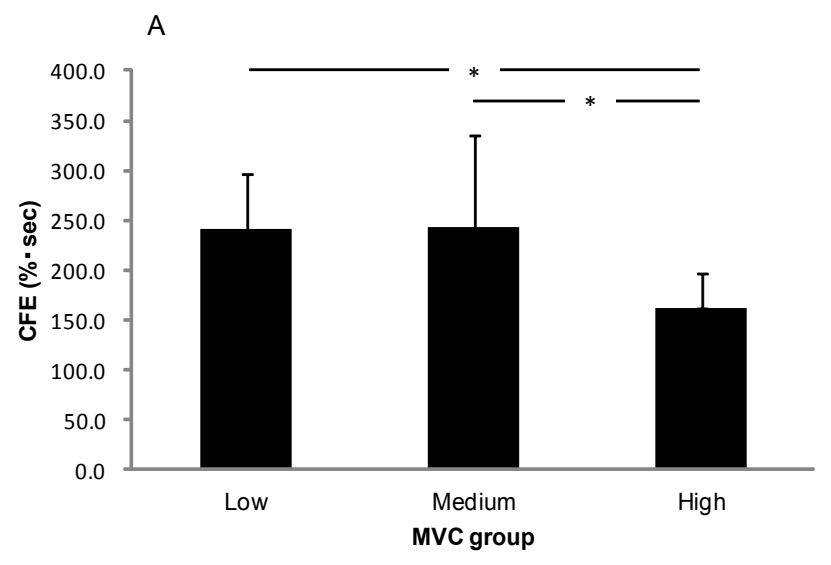

B

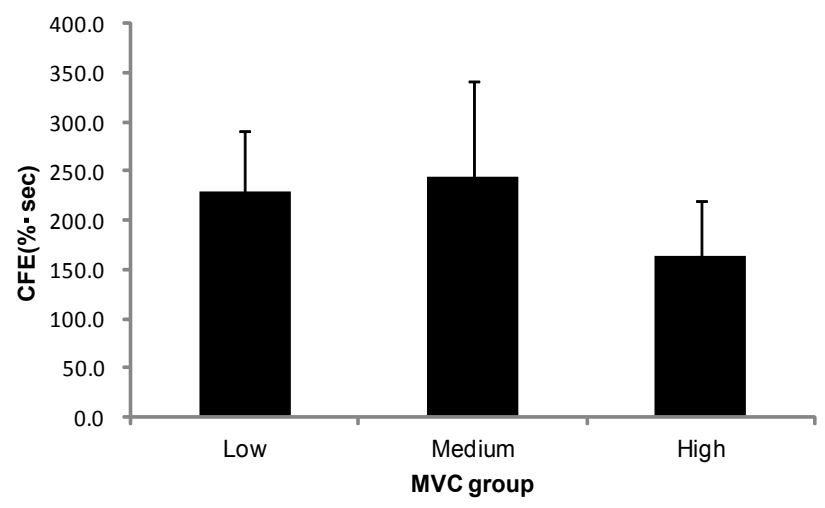

Figure 2. Mean values of controlled force exertion (CFE) (note) A: 5-15\% MVC phase, B: 15--25\% MVC phase; Low: MVC < 388.1N; Medium: $388.1 \mathrm{~N} \leqq \mathrm{MVC}<523.3 \mathrm{~N} ;$ High: $\mathrm{MVC} \geqq 523.3 \mathrm{~N}$ ) 


\section{Results}

Figure 2 shows the means and the standard deviations for the measured CFE values in both low demand value (5\%-15\% MVC) and high demand value (15\%-25\% MVC) phases of the three groups with different grip strengths. Table 2 shows the results of one-way analysis of variance and the measured CFE values in the three groups. Significant differences for the measured CFE values in the three groups were found only in the low demand value phase; the values for the high grip strength group were lower than those of the low and medium grip strength groups. Significant correlations were found between CFE measured value and grip strength in low $(\mathrm{r}=-0.24$; $\mathrm{p}=0.08)$ and in high $(\mathrm{r}=-0.32, \mathrm{p}=0.02)$ demand value phases, but both correlations showed a low value (determination coefficient: below 10.2\%).

Table 2. The results of analysis of variance (ANOVA)

\begin{tabular}{lllllll}
\hline \multirow{3}{*}{ Factor } & \multicolumn{5}{c}{ ANOVA } \\
\cline { 2 - 6 } & & $d f$ & $M S$ & $F$ & $\eta^{2}$ & $p$ \\
\hline
\end{tabular}

CFE in $5-15 \%$ MVC phase

$\begin{array}{cccccc}\text { MVC group } & 2 & 21889.2 & 3.43 & 0.12 & 0.04 \\ \text { Error } & 51 & 6374.7 & & & \\ \text { Total } & 53 & & & & \end{array}$

CFE in $15-25 \%$ MVC phase

\begin{tabular}{cccccc} 
MVC group & 2 & 21224.3 & 2.97 & 0.10 & 0.06 \\
Error & 51 & 7156.8 & & & \\
Total & 53 & & & & \\
\hline
\end{tabular}

note) MS: mean square.

\section{Discussion}

The functional role related to movement performance may differ for each body part that controls individual movements. The basal ganglia, particularly the striatonigral system, and cerebellum are generally considered to be associated with skilled motor movements. The CFE test uses visual tracking with a sinusoidal waveform display providing visual feedback regarding grip exertion errors and feed-forward strategies, such as anticipatory motion [1]. The performance of this test necessitates excellent hand-eye coordination (grip force) that responds to feedback, such as force exertion sense and visual target matching. Therefore, the abovementioned visual information processing and motor control functions are considered to largely reflect measured CFE values.

In this study, we hypothesized that the effect of MVC on the measured CFE value differs in the low demand value $(5 \%-15 \%$ of $\mathrm{MVC})$ and high demand value (15\%-25\% of MVC) phases in the CFE test; therefore, we examined the difference of CFE measured values in three groups with different MVCs (mean grip strength, 457.1 N), in addition to the relationship between MVC and the measured value in both phases.

In this study, the measured value showed a significant difference only in the low demand value phase, and the value in the group with the largest grip strength was smaller than the values seen in the other two groups. However, the effect size $\left(\eta^{2}\right)$ was low $\left(\eta^{2}=0.12\right)$ and no significant difference was found between the low grip strength (mean grip strength $363.9 \mathrm{~N}$ ) and the medium grip strength (mean grip strength $468.2 \mathrm{~N}$ ) groups.

Previous reports $[10,22,23]$ have shown that the control of a subject's force output differs in the ascending and decreasing phases. The amplitude of motor-evoked potentials, which change in response to force, also reportedly differs between the two phases [24]. In the ascending and decreasing demand value phases, exerted style (contraction and relaxation of muscle) reflects the measured value compared with the degree of exertion (greatness); however, maximal grip strength is considered to relate primarily to the measured CFE value in both phases because the degree of exertion differs. In the low grip strength and high grip strength groups, the mean values of grip strength were $363.9 \mathrm{~N}$ and $562.7 \mathrm{~N}$, respectively, and the former was approximately half of grip strength. This suggests that the grip strengths exerted by the former and the latter groups were 18.2-54.6N and $28.1-84.4 \mathrm{~N}$, respectively, in the low demand value phase $(5 \%-15 \%$ of MVC), whereas that in the high demand value phase (15\%-25\% of MVC) were 54.6-91.0 $\mathrm{N}$ and 84.4-140.7 N, respectively. Choi et al. [25] reported that the effect of grip strength on the error between pursuing the target (demand value) and the exerted value was larger at low demand value phase, but small at demand values over $15 \%$ MVC. The hypothesis of this study was not supported in the high demand value phase. Although it was partly supported in the low demand value phase, the effect size was low and there was no significant difference between the low and medium grip strength groups. Therefore, we consider that the difference of maximum grip strength has a negligible effect on the measured value.

On the other hand, grip strength was significantly related to the measured value only in the high demand value phase, but the determination coefficient (approximately $10 \%$ ) were low. In this study, we hypothesized that if MVC affects the measured CFE value, the relationship between MVC and the measured value differs in both phases. As the relationship was found only in the high demand value phase and not in the low demand value phase, the hypothesis in this study was supported. However, the contribution of grip strength to the measured value was very low in the high demand value phase and the difference in the determination coefficient in both phases was only close to $4 \%(5.76 \%$ vs $10.24 \%)$. Bemben et al. [26] reported that the peripheral muscle activity of young participants in maximal muscle exertion is superior to that of older participants. According to Voelcker-Rehage \& Alberts [27], young participants are better at performing the changing force-tracking task because they have superior peripheral muscular responses to the changing target and exertion of coordinated neuromuscular function. They can control their exertion of grip strength in both phases of the low and high demand value, irrespective of superior or inferior grip strength. Therefore, the difference of maximum grip strength in both phases is inferred to have a negligible effect.

The participants in the present study were healthy male adults with a mean maximal grip strength greater than 
457.1N. In middle-aged adults, who have inferior peripheral muscular responses to the changing target and exertion of coordinated neuromuscular function, the relationship between the measured CFE value and grip strength may differ from that in young adults. The CFE test is useful in evaluating the CFE in middle-aged adults because of the use of submaximal grip control. A follow-up study will be required to examine the problems identified in this study in relation to the middle-aged people.

In conclusion, it is judged that the difference in maximum grip strength has a negligible effect on the measured CFE value in both low demand value and high demand value phases of the CFE test in young males.

\section{Acknowledgments}

This study was supported in part by a grant-in-aid for Scientific Research (project number 20500506) to Y. Nagasawa from the Ministry of Education, Science and Culture of Japan.

\section{References}

[1] Ofori, E., Samson, J. M., and Sosnoff, J. J. (2010). Age-related differences in force variability and visual display. Explain Brain Research, 203, 299-306.

[2] Deutsch, K. M., and Newell, K. M. (2001). Age differences in noise and variability of isometric force production. Journal of Experimental Child Psychology, 80, 392-408.

[3] Galganski, M. E., Fuglevand, A. J., and Enoka, R. M. (1993). Reduced control of motor output in a human hand muscle of elderly subjects during submaximal contractions. Journal of Neurophysiology, 69, 2108-2115.

[4] Sosnoff, J. J., and Newell, K. M. (2008). Age-related loss of adaptability to fast time scales in motor variability. Journal of Gerontology B: Psychological Science and Social Science, 63, 344-352.

[5] Henatsch, H-D., and Langer, H. H. (1985). Basic neurophysiology of motor skills in sport: a review. International Journal of Sports Medicine, 6, 2-14.

[6] Nagasawa, Y., and Demura, S. (2002). Development of an apparatus to estimate coordinated exertion of force. Perceptual and Motor Skills, 94, 899-913.

[7] Brown, S. W., and Bennett, E. D. (2002). The role of practice and automaticity in temporal and nontemporal dual-task performance. Psychological Research, 66, 80-89.

[8] Nagasawa, Y., Demura, S., Yamaji, S., Kobayashi, H., and Matsuzawa, J. (2000). Ability to coordinate exertion of force by the dominant hand: comparisons among university students and 65- to 78- year-old men and women. Perceptual and Motor Skills, 90, 995-1007.

[9] Shechtman, O., Sindhu, B.S., Davenport P, W. (2012). Using the "visual target grip test" to identify sincerity of effort during grip strength testing. Journal of Hand Therapy, 25, 320-329.

[10] Kubota, H., and Demura, S. (2015). Adjustment errors in ascending and descending phases of target level in controlled force exertion. Perceptual and Motor Skills, 121, 613-620.
[11] Demura, S., Yamaji, S., Goshi, F., and Nagasawa, Y. (2001). Lateral dominance of legs in maximal muscle power, masculer endurance, and grading ability. Perceptual and Motor Skills, 93, 11-23.

[12] Bemben, M.G., Massey, B.H., Bemben, D.A., Misner, J.E., and Boileau, R.A. (1991). Isometric muscle force production as a function of age in healthy 20- to 74-yr.-old men. Medicine and Science in Sports and Exercise, 23, 1302-1310.

[13] Ahrenfeldt, L.J., Scheel-Hincke, L.L., Kjærgaard, S., Möller, S., Christensen, K., and Lindahl-Jacobsen, R. (2018). Gender differences in cognitive function and grip strength: a cross-national comparison of four European regions. European Journal of Public Health. 2018 Dec 24.

[14] Demura, S., Sato, S., and Nagasawa, Y. (2009). Re-examination of useful items for determining hand dominance. Gazzeta Medica Italiana - Archives of Science Medicine, 168, 169-177.

[15] Society for Physical Fitness Standards Research in Tokyo Metropolitan University. (Ed.) (2000). [New Physical Fitness Standards of Japanese People]. (pp. 20-85). Tokyo, Japan: Fumaido. [in Japanese]

[16] Walamies, M., and Turjanmaa, V. (1993). Assessment of the reproducibility of strength and endurance handgrip parameters using a digital analyzer. European Journal of Applied Physiology and Occupational Physiology, 67, 83-86.

[17] Skelton, D. A., Greig, C. A., Davies, J. M., and Young, A. (1994). Strength, power and related functional ability of healthy people aged 65-89 years. Age and Ageing, 23,371-377.

[18] Nagasawa, Y., Demura, S., and Nakata, M. (2003). Reliability of a computerized target-pursuit system for measuring coordinated exertion of force. Perceptual and Motor Skills, 96, 1071-1085.

[19] Nagasawa, Y., and Demura, S. (2009). Age and sex differences of controlled force exertion measured by a computer-generated sinusoidal target-pursuit system. Journal of Physiological Anthropology, 28, 199-205.

[20] Nagasawa, Y., Demura, S., and Kitabayashi, T. (2004). Concurrent validity of tests to measure the coordinated exertion of force by computerized target-pursuit. Perceptual and Motor Skills, 98: 551-560.

[21] Nagasawa, Y., and Demura, S. (2016) Effect of Short-Term Exercise on Controlled Force Exertion in Young and Middle-Aged Adults. American Journal of Sports Science and Medicine, 4, 78-82.

[22] Gorassini, M., Yang, J. F., Siu, M., and Bennett, D. J. (2002) Intrinsic activation of human motoneurons: possible contribution to motor unit excitation. Journal of neurophysiology, 87 , 1859-1866.

[23] Romaiguere, P., Vedel, J. P., and Pagni, S. (1993). Comparison of fluctuations of motor unit recruitment and de-recruitment thresholds in man. Experimental Brain Research, 95, 527-522.

[24] Kimura, T., Yamanaka, K., Nakazawa, K., Miyoshi, T., Akai, M., and Ohtsuki, T. (2003). Hysteresis in corticospinal excitability during gradual muscle contraction and relaxation in humans. Experimental Brain Research, 152, 123-132.

[25] Choi, K-H., Kim, D-M., Lee, S-Y., Lee, J-H., and Kong Y-K. (2017). Evaluation of the controlled grip force exertion tasks associated with age, gender, handedness and target force level. International Journal of Occupatinal Safety and Ergonomics, May 23: $1-9$.

[26] Bemben, M.G., Massey, B.H., Bemben, D.A., Misner, J.E. and Boileau, R.A. (1996). Isometric intermittent endurance of four muscle groups in men aged 20-74 yr. Medicine and Science in Sports and Exercise, 28, 145-154.

[27] Voelcker-Rehage, C., and Alberts. J.L. (2005). Age-related changes in grasping force modulation. Experimental Brain Research, 166, 61-70. 